\title{
Hierarchical Control of Redundant Aerial Manipulators with Enhanced Field of View
}

\author{
Andre Coelho ${ }^{1,2}$, Yuri S. Sarkisov ${ }^{1,4}$, Jongseok Lee ${ }^{1}$, Ribin Balachandran ${ }^{1}$, \\ Antonio Franchi ${ }^{2,3}$, Konstantin Kondak ${ }^{1}$, Christian Ott ${ }^{1}$
}

\begin{abstract}
Providing the operator with a good view of the remote site is of paramount importance in aerial telemanipulation. In light of that, this paper proposes the application of a hierarchical control framework in order to tackle the problem of adjusting the field of view of an on-board camera as a secondary task. The proposed approach ensures that the flying base, and consequently the camera, can be steered in order to provide a distant operator with a desired field of view without disturbing the end-effector pose. The approach is focused on aerial manipulators with torque-controlled arms, like the DLR Suspended Aerial Manipulator (SAM), while allowing the base to be directly torque-controlled or, alternatively, through an inner-loop velocity controller. Quantitative, qualitative, and real-scenario experimental validation is carried out using the SAM and confirms the need for such an approach and its efficacy in achieving decoupled field-of-view control.
\end{abstract}

\section{INTRODUCTION}

Intensive technology development in the field of aerial manipulation has paved the way towards the application of aerial robots to more complex interaction tasks. Among others, preliminary results have demonstrated the applicability of such systems in the areas of maintenance and inspection of power lines [1], [2], bridges [3], [4] and industrial-plant pipelines [5], [6].

As the environment gets less structured and more dynamic, the introduction of a human operator to the loop in a teleoperation or shared-control fashion can facilitate the fulfillment of more complex aerial manipulation tasks [7]-[9]. In such applications, the operator strongly relies on visual information provided by on-board sensors and cameras, as direct visual contact is rarely possible. Our recent work introduced a visual-inertial framework for aerial telemanipulation [9], where, in addition to haptic information, the operator is provided with visual feedback through direct stream from an on-board camera and a 3D virtual reality scene. The pose of the end-effector and of the objects of interest is updated in real-time based on joint-position measurements and a marker-detection algorithm. An overview of the proposed framework can be seen in Fig. 1. The operator, sitting in the ground station, relies on visual information in order to command the remotely-located DLR Suspended Aerial

\footnotetext{
${ }^{1}$ The authors are with the Institute of Robotics and Mechatronics of the German Aerospace Center (DLR), Oberpfaffenhofen, Germany.

${ }^{2}$ The authors are with the Robotics and Mechatronics Lab, Faculty of Electrical Engineering, Mathematics \& Computer Science, University of Twente, Netherlands.

${ }^{3}$ The author is with LAAS-CNRS, University of Toulouse, France.

${ }^{4}$ The author is with Space CREI, Skolkovo Institute of Science and Technology (Skoltech), Moscow, Russia.

Andre.Coelhoddlr.de
}

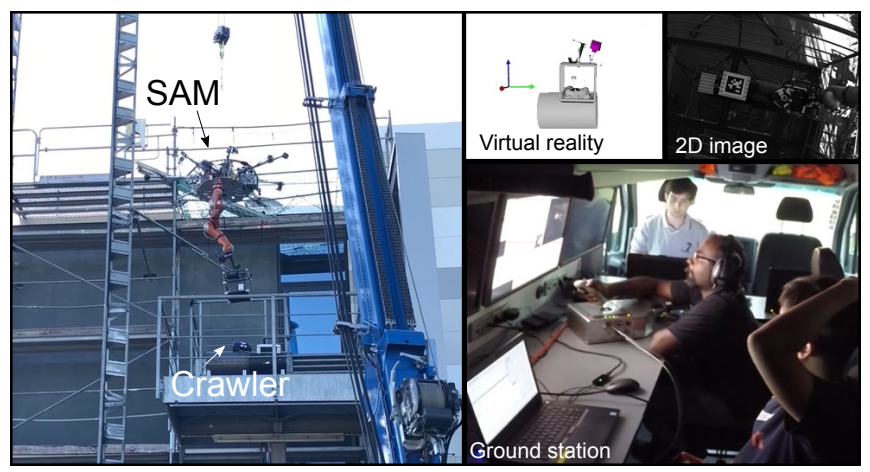

Fig. 1: Visual-inertial teleoperation framework [9]. In addition to haptic feedback, the operator is provided with visual information through a virtual-reality environment as well as raw images from a camera.

Manipulator (SAM) [10] using a haptic device. The vision system relies on two cameras, namely, a hand-eye camera attached to the end-effector and an eye-to-hand camera attached to the UAV base (see Fig. 2).

Although the proposed framework allowed a real teleoperation task to be fulfilled, namely the deployment and recovery of an inspection crawler, some issues in the visualization system were identified:

(a) eventual occlusion of the markers in the eye-to-hand camera image;

(b) poor view of the task in the streamed image; and

(c) mismatch between arm and joystick motions in the streamed image.

The first issue, (a), has been tackled in our previous work [9] by relying solely on the hand-eye camera for marker detection, which might decrease the robustness of the system. Issues (b) and (c), on the other hand, still need to be tackled. Issue (b) means that it can be hard to recognize objects in the scene in case the camera is not in a favorable pose. Respectively, (c) occurs when, due to the orientation of the camera, the directions of motion of the joystick map to different (non-orthogonal) directions of motion of the end-effector in the image, which makes it less intuitive for the operator to command the arm to a desired pose. To understand this problem, some readers might recall the feeling of playing a video game and suddenly having the controls inverted.

In order to solve the aforementioned issues (b) and (c) and propose a more robust solution to (a) by also enabling the use of the eye-to-hand camera for marker detection, we aim 
at endowing the SAM with the capability of moving its base in order to change the field of view (FoV) of the eye-to-hand camera during operation. In order to do so, two additional requirements have to be accounted for. First, despite being part of the kinematic chain of the robot, the base motion should not undesirably disturb the end-effector motion, i.e., the FoV-adjustment task should occur in the null space [11] of the end-effector motion. In addition, the developed control method should allow for independent velocity control of the base in a cascaded manner. This is owes to the fact that the SAM is coped with combined actuation of the base, composed of propellers and variable-length cables [10] with the latter being commanded by winch motors with their own velocity controller. Therefore, it is meaningful to have some degrees of freedom (DoF) of the desired base trajectory tracked by an inner-loop velocity controller, which takes both propellers and winches into account.

A key characteristic of the SAM, which allows for the fulfilling of the aforementioned requirements is its kinematic redundancy. By having more DoF than the minimum required, whole-body control techniques can be applied to make the aerial robot perform the main task in an optimal manner or to accomplish additional subtasks without disturbing the main one. A possible way of achieving a desired FoV while successfully accomplishing the manipulation task is by designing a hierarchical controller, which has the camera pose as a secondary task to be performed. A similar idea was presented in [12] and [13]. It was shown that adding the camera placement as a lower-priority task in a hierarchicalcontrol structure allows for the successful completion of aerial visual-servoing tasks. In those works, the redundancy is solved at kinematic level, i.e., by providing a desired velocity to the secondary task, which is in the null space of the primary one. Such controllers are based on the assumption that a desired velocity would be perfectly followed by the manipulator joints, which may or may not hold, based on task and hardware specifications. Nevertheless, since recently developed aerial manipulators [10], [14] are composed of torque controlled manipulators, dynamical decoupling [15] of the redundant robot can be ensured in order to further improve the performance of the task.

Null-space FoV control is a crucial add-on for both autonomous tasks, as in [14] and [16], and teleoperation, as in [17] and [18]. In contrast to our previous works [17], [18], which mainly focused on the passivity and stability implications of performing multi-task aerial telemanipulation, this paper is intended to be more experimental, aiming at analyzing the performance of the hierarchical control itself as well as its applicability to real scenarios, including not only telemanipulation, but also vision-based autonomous manipulation, also considering the case where the base velocity is controlled by an inner-loop controller has not yet been tested.

In summary, this paper focuses on the application of a dynamically-decoupling control approach in order to achieve multi-task aerial manipulation, where the flying base is force or velocity controlled to achieve a desired camera view with- out disturbing the end-effector pose. The proposed approach is an important add-on to the visual-inertial teleoperation framework presented in [9], as it endows the operator with the capability of adjusting the camera view to avoid marker occlusions and motion mismatch between joystick and arm. In addition, it prepares the SAM for the operation of the winches by enabling the base to be controlled by an innerloop velocity controller. The performance of the proposed method is demonstrated through experiments performed on the SAM.

The remainder of this paper is structured as follows. Section II provides relevant details about the Suspended Aerial Manipulator while Section III describes the control approach designed to accomplish both manipulation and FoV control with the possibility of having an inner-loop control of the base velocity. Moreover, Section IV presents both quantitative and qualitative experimental validation, as well as results from the application of the proposed approach to fulfill a peg-in-hole task. Finally, Section V concludes the paper.

\section{The Suspended Aerial Manipulator}

The DLR Suspended Aerial Manipulator, also known as SAM (illustrated in Fig. 2), was developed in the scope of the AEROARMS project [8] with the purpose of allowing teleoperated inspection and manipulation tasks in industrial scenarios. A description of the relevant details of the system is as follows. The flying platform is equipped with eight propellers, capable of providing omni-directional wrenches for the actuation of the floating base. An industrial robotic arm, KUKA LWR (a 7-DoF torque-controlled manipulator), is attached to the platform for performing aerial manipulation tasks. Moreover, the platform is suspended through a set of winch-actuated cables, whose velocity is independently controlled. Furthermore, an industrial monocular camera (Allied Vision Mako) mounted on the platform provides highresolution images, which enables perceiving great part of the operational space of the manipulator and can be used for object tracking, as an example. The SAM can be carried by a manned/unmanned helicopter as well as an indoor/outdoor crane. For the purpose of this work, both an overhead crane and a mobile one were chosen as carriers.

For more information about the constructive characteristics of the SAM, the reader is referred to [10].

\section{HiERARCHICAL MANipUlation AND FoV CONTROLler}

For the purpose of controlling the FoV of the eye-to-hand camera of the SAM, which is attached to the flying base, it is considered sufficient to perform a regulation task in yaw, i.e., rotation around its suspension axis at rest (inertial z-axis), while roll and pitch are assumed to be successfully damped by an oscillation-damping controller [19]. In fact, as will be shown in Section IV, during nominal motion of the arm (i.e., avoiding excessive stretching) and with damped oscillations, the roll and pitch motions of the platform are only slightly affected. Based on that, the aerial system can be regarded as 


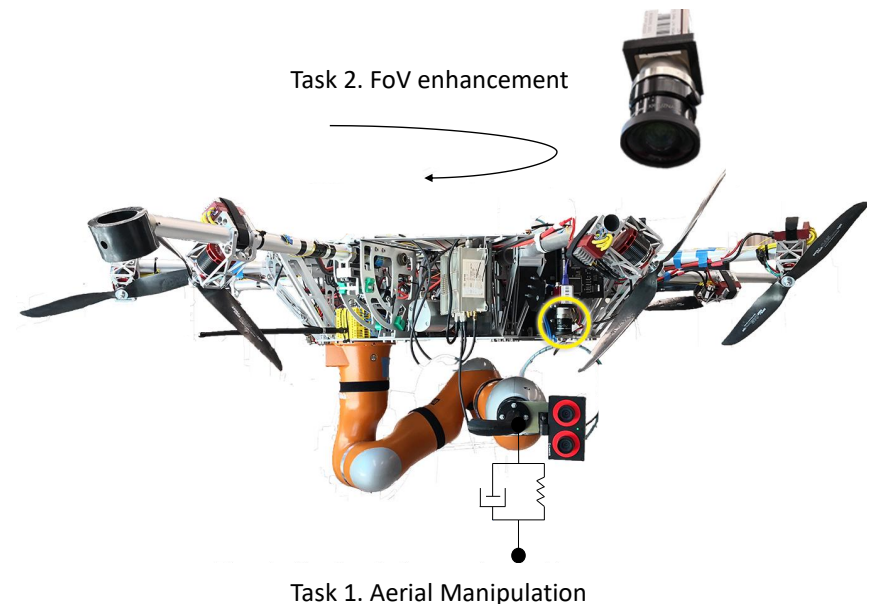

Fig. 2: Proposed framework. The primary task is relative to the pose of the end-effector. The secondary one consists of enhancing the FoV of the eye-to-hand camera, circled in yellow and depicted in the top-right corner.

having eight DoF, i.e., yaw rotation of the flying base and the seven DoF of the manipulator. Based on that assumption, the camera task pose will solely depend on the yaw motion and can be defined as a regulation controller along that DoF. On the other hand, the manipulation task depends on the eight joints and may also command the yaw motion in order to achieve a desired pose. Nevertheless, a hierarchical controller can be applied in order to ensure that the main task will only make use of the yaw motion when strictly necessary, while camera pose regulation will be fulfilled as long as it does not disturb the main task (see Fig. 2). Additionally, in order to completely allocate the DoFs in the control design and avoid undesirable internal motions, the elbow joint of the arm will be damped as a third task. The details about the implementation of such a controller will be provided in the following sections.

\section{A. Dynamics of the redundant aerial manipulator}

Based on the aforementioned damped-oscillation assumption, the dynamics of the SAM can be written as

$$
\boldsymbol{M}(\boldsymbol{q}) \ddot{\boldsymbol{q}}+\boldsymbol{C}(\boldsymbol{q}, \dot{\boldsymbol{q}}) \dot{\boldsymbol{q}}+\boldsymbol{G}(\boldsymbol{q})=\tau+\tau_{\text {ext }},
$$

where $\boldsymbol{q} \in \mathbb{R}^{8}$ is a set of generalized coordinates corresponding to the yaw angle of the base and the manipulator joint angles, $\boldsymbol{M}(\boldsymbol{q}) \in \mathbb{R}^{8 \times 8}$ is the inertia matrix, $\boldsymbol{C}(\boldsymbol{q}, \dot{\boldsymbol{q}}) \dot{\boldsymbol{q}} \in \mathbb{R}^{8}$ is a vector of Coriolis and centrifugal forces, and $\boldsymbol{G}(\boldsymbol{q}) \in$ $\mathbb{R}^{8}$ is the gravitational generalized torque vector. $\tau \in \mathbb{R}^{8}$ corresponds to the control torque applied around the yaw axis and to the manipulator joint torques. $\tau_{e x t} \in \mathbb{R}^{8}$ is the reflection of external forces and torques to the yaw and manipulator joints.

The task velocities of the three tasks to be controlled are defined as

$$
\begin{aligned}
& \boldsymbol{V}_{1}=\boldsymbol{J}_{1}(\boldsymbol{q}) \dot{\boldsymbol{q}} \\
& \boldsymbol{V}_{2}=\dot{q}_{1}=\boldsymbol{J}_{2}(\boldsymbol{q}) \dot{\boldsymbol{q}} \\
& \boldsymbol{V}_{3}=\dot{q}_{4}=\boldsymbol{J}_{3}(\boldsymbol{q}) \dot{\boldsymbol{q}}
\end{aligned}
$$

where $V_{1} \in \mathbb{R}^{6}$ is the body velocity of the end-effector, $V_{2} \in \mathbb{R}$ is the rotational velocity of the platform around its suspension axis, and $V_{3} \in \mathbb{R}$ is the velocity of the elbow joint of the arm. Moreover, $\boldsymbol{J}_{1}(\boldsymbol{q}) \in \mathbb{R}^{6 \times 8}, \boldsymbol{J}_{2}(\boldsymbol{q}) \in \mathbb{R}^{1 \times 8}$, and $\boldsymbol{J}_{3}(\boldsymbol{q}) \in \mathbb{R}^{1 \times 8}$ are the Jacobian matrices that map joint velocities to task velocities.

In order to perform hierarchical control, the framework presented in [20] will be followed, which defines an augmented Jacobian as

$$
\boldsymbol{J}_{i}^{\text {aug }}(\boldsymbol{q})=\left[\begin{array}{lll}
\boldsymbol{J}_{1}(\boldsymbol{q})^{T} & \ldots & \boldsymbol{J}_{i}(\boldsymbol{q})^{T}
\end{array}\right]^{T}, \quad i \in\{1,2,3\},
$$

where $\boldsymbol{J}_{3}^{\text {aug }}(\boldsymbol{q})$ is assumed to keep full row rank during the performed tasks. Furthermore,

$$
\boldsymbol{V}=\left[\begin{array}{lll}
\boldsymbol{V}_{1}^{T} & \boldsymbol{V}_{2} & \boldsymbol{V}_{3}
\end{array}\right]^{T}=\boldsymbol{J}_{3}^{\mathrm{aug}}(\boldsymbol{q}) \dot{\boldsymbol{q}},
$$

where $\boldsymbol{V} \in \mathbb{R}^{8}$ is the augmented task velocity, composed of all velocities stacked as a vector.

In order to define a set of decoupled velocity coordinates, where lower-priority velocities and accelerations do not disturb higher-priority ones, dynamically consistent null-space projection matrices (see [20], [21]) can be defined as

$$
\boldsymbol{N}_{i}(\boldsymbol{q})= \begin{cases}\boldsymbol{I}, & \text { for } i=1 \\ \boldsymbol{I}-\boldsymbol{J}_{i-1}^{\mathrm{aug}}(\boldsymbol{q})^{T} \boldsymbol{J}_{i-1}^{\mathrm{aug}}(\boldsymbol{q})^{M+, T}, & \text { for } i \in\{2,3\},\end{cases}
$$

where $\boldsymbol{I}$ is the identity matrix and $(\cdot)^{M+}$ represents the inertia-weighted pseudoinverse [11] of the augmented Jacobian matrix. Using the aforementioned projections, the following decoupling Jacobian matrices are defined.

$$
\overline{\boldsymbol{J}}_{i}(\boldsymbol{q})=\boldsymbol{J}_{i}(\boldsymbol{q}) \boldsymbol{N}_{i}(\boldsymbol{q})^{T},
$$

such that a set of decoupled task velocities is defined as

$$
\underbrace{\left[\begin{array}{l}
\mathcal{V}_{1} \\
\mathcal{V}_{2} \\
\mathcal{V}_{3}
\end{array}\right]}_{\mathcal{V}}=\underbrace{\left[\begin{array}{l}
\overline{\boldsymbol{J}}_{1}(\boldsymbol{q}) \\
\overline{\boldsymbol{J}}_{2}(\boldsymbol{q}) \\
\overline{\boldsymbol{J}}_{3}(\boldsymbol{q})
\end{array}\right]}_{\overline{\boldsymbol{J}}(\boldsymbol{q})} \dot{\boldsymbol{q}},
$$

where $\mathcal{V}$ is the augmented decoupled task velocity and $\overline{\boldsymbol{J}}(\boldsymbol{q})$ is the Jacobian that maps joint velocities to $\mathcal{V}$.

By using (9) and its time derivative, the robot dynamics (1) are transformed into

$$
\boldsymbol{\Lambda}(\boldsymbol{q}) \dot{\mathcal{V}}+\mu(\boldsymbol{q}, \dot{\boldsymbol{q}}) \mathcal{V}=\overline{\boldsymbol{J}}(\boldsymbol{q})^{-T}\left(\tau-\tau_{\text {ext }}-\boldsymbol{G}(\boldsymbol{q})\right),
$$

where

$$
\boldsymbol{\Lambda}(\boldsymbol{q})=\overline{\boldsymbol{J}}^{-T} \boldsymbol{M} \overline{\boldsymbol{J}}^{-1}=\operatorname{blkdiag}\left(\boldsymbol{\Lambda}_{1}(\boldsymbol{q}), \ldots, \boldsymbol{\Lambda}_{r}(\boldsymbol{q})\right),
$$

and

$$
\begin{aligned}
\mu(\boldsymbol{q}, \dot{\boldsymbol{q}}) & =\overline{\boldsymbol{J}}^{-T}\left(\boldsymbol{C}-\boldsymbol{M} \overline{\boldsymbol{J}}^{-1} \dot{\overline{\boldsymbol{J}}}\right) \overline{\boldsymbol{J}}^{-1} \\
& =\left[\begin{array}{lll}
\mu_{1,1}(\boldsymbol{q}, \dot{\boldsymbol{q}}) & \mu_{1,2}(\boldsymbol{q}, \dot{\boldsymbol{q}}) & \mu_{1,3}(\boldsymbol{q}, \dot{\boldsymbol{q}}) \\
\mu_{2,1}(\boldsymbol{q}, \dot{\boldsymbol{q}}) & \mu_{2,2}(\boldsymbol{q}, \dot{\boldsymbol{q}}) & \mu_{2,3}(\boldsymbol{q}, \dot{\boldsymbol{q}}) \\
\mu_{3,1}(\boldsymbol{q}, \dot{\boldsymbol{q}}) & \mu_{3,2}(\boldsymbol{q}, \dot{\boldsymbol{q}}) & \mu_{3,3}(\boldsymbol{q}, \dot{\boldsymbol{q}})
\end{array}\right]
\end{aligned}
$$


It can be noted that, due to the decoupling transformation, a block diagonal inertia matrix $\boldsymbol{\Lambda}(\boldsymbol{q}) \in \mathbb{R}^{8 \times 8}$ is achieved, which ensures no direct kinetic energy transfer among tasks. On the other hand, the transformed Coriolis matrix $\mu(\boldsymbol{q}, \dot{\boldsymbol{q}}) \in$ $\mathbb{R}^{8 \times 8}$ is usually fully occupied, allowing power-conserving inter-task interference at velocity level.

\section{B. Hierarchically-decoupling controller}

The first step to completely decouple the dynamics of the tasks is performing a preliminary compensation action, which cancels out the cross-coupling and compensates for gravity, as follows.

$$
\begin{aligned}
\tau & =\tau_{c}+\tau_{\mu}+\boldsymbol{G}(\boldsymbol{q}) \\
\tau_{\mu} & =\sum_{i=1}^{r}\left(\overline{\boldsymbol{J}}_{i}^{T}\left(\sum_{j=1}^{i-1} \mu_{i, j} \mathcal{V}_{j}+\sum_{j=i+1}^{r} \mu_{i, j} \mathcal{V}_{j}\right)\right),
\end{aligned}
$$

where $\mu_{i, j}$ corresponds to the $\tau_{\mu}$ is responsible for compensating for the cross-coupling terms in $\mu(\boldsymbol{q}, \dot{\boldsymbol{q}})$ and making it block diagonal. In turn, $\tau_{c}$ is the effective control signal, after compensation, which will be responsible for accomplishing the desired tasks in a hierarchical manner. By applying the pre-compensation terms in (13), the dynamics of the system in the decoupled task velocities becomes

$$
\boldsymbol{\Lambda}_{i}(\boldsymbol{q}) \dot{\mathcal{V}}_{i}+\mu_{i}(\boldsymbol{q}, \dot{\boldsymbol{q}}) \mathcal{V}_{i}=\mathcal{F}_{c, i}+\mathcal{F}_{\text {ext }, i}, \quad i \in\{1,2,3\}
$$

where $\mathcal{F}_{c, i}=\overline{\boldsymbol{J}}_{i}(\boldsymbol{q})^{-T} \tau_{c, i}$ and $\mathcal{F}_{\text {ext }, i}=\overline{\boldsymbol{J}}_{i}(\boldsymbol{q})^{-T} \tau_{\text {ext }, i}$.

In order to control the pose of the end-effector (as a primary task) and of the flying base (as a secondary task), the following error elements can be defined

$$
\begin{aligned}
& \boldsymbol{g}_{\mathrm{e}, 1}=\boldsymbol{g}_{\text {des }, 1}^{-1} \boldsymbol{g}_{1}=\left(\boldsymbol{R}_{\mathrm{e}, 1}, \boldsymbol{p}_{\mathrm{e}, 1}\right), \\
& \boldsymbol{g}_{\mathrm{e}, 2}=\boldsymbol{g}_{2, \text { des }}^{-1} \boldsymbol{g}_{2}=\left(\boldsymbol{R}_{\mathrm{e}, 2}, \boldsymbol{p}_{\mathrm{e}, 2}\right),
\end{aligned}
$$

where $\boldsymbol{g}_{\text {des }, 1}, \boldsymbol{g}_{1}, \boldsymbol{g}_{\text {des }, 2}$, and $\boldsymbol{g}_{2}$ are homogeneous transformation matrices on $S E(3)$, which describe the desired and current poses of the end-effector (subscript 1) and of the flying base (subscript 2) with respect to a fixed inertial frame.

To accomplish the desired tasks, the body control wrench $\mathcal{F}_{c, 1}$ and the control torques $\mathcal{F}_{c, 2}$ and $\mathcal{F}_{c, 3}$ can be defined as follows [22], [23].

$$
\begin{aligned}
& \mathcal{F}_{c, 1}=\left[\begin{array}{c}
-\boldsymbol{R}_{\mathrm{e}, 1}^{T} \boldsymbol{K}_{P x} \boldsymbol{p}_{\mathrm{e}, 1} \\
-2 \boldsymbol{R}_{\mathrm{e}, 1}^{T} \boldsymbol{E}\left(\eta_{e, 1}, \varepsilon_{e, 1}\right)^{T} \boldsymbol{K}_{O x} \varepsilon_{e, 1}
\end{array}\right]-\boldsymbol{K}_{D x} \boldsymbol{V}_{1}, \\
& \mathcal{F}_{c, 2}=-2 \boldsymbol{S}\left(\boldsymbol{R}_{\mathrm{e}, 2}^{T} \boldsymbol{E}\left(\eta_{e, 2}, \varepsilon_{e, 2}\right)^{T} \boldsymbol{K}_{O b} \varepsilon_{e, 2}-\boldsymbol{K}_{D b} \boldsymbol{V}_{2}\right) \\
& \mathcal{F}_{c, 3}=-K_{D 3} \boldsymbol{V}_{3}
\end{aligned}
$$

where the matrices $\boldsymbol{K}_{(.)}$are positive definite gain matrices, $K_{D 3}$ is a scalar damping gain, and $\boldsymbol{S}=\left[\mathbf{0}_{1 \times 2} 1\right]$ is a selection matrix that selects the $\mathrm{z}$ component of the control torque. Moreover, $\eta_{e, i}$ and $\varepsilon_{e, i}$ are the scalar and vector parts of a quaternion representation of $\boldsymbol{R}_{e, i}$, respectively, and $\boldsymbol{E}\left(\eta_{e, i}, \varepsilon_{e, i}\right)=\eta_{e, i} \boldsymbol{I}_{3}-\widehat{\varepsilon}_{e, i}$, for $i \in\{1,2\}$.

To achieve the desired coordinated motion of base and end-effector, $\mathcal{F}_{c}=\left[\begin{array}{lll}\mathcal{F}_{c, 1}^{T} & \mathcal{F}_{c, 2} & \mathcal{F}_{c, 3}\end{array}\right]^{T}$ is transformed into $\tau_{c}$ through $\overline{\boldsymbol{J}}(\boldsymbol{q})^{T}$ and plugged into (13).
By applying the proposed control law, in the absence of external forces, asymptotic stability of $\boldsymbol{g}_{\mathrm{e}, 1}=\boldsymbol{I}$ and conditional stability (stable behavior once the higher-priority task has converged) of $\boldsymbol{g}_{\mathrm{e}, 2}=\boldsymbol{I}$ task are achieved. Moreover, convergence of $\boldsymbol{g}_{\mathrm{e}, 2}=\boldsymbol{I}$ is also achieved in case there is no conflict between tasks, as is the case for the desired application. For more details, see [20].

If the aerial manipulator is to be directly torque controlled, the aforementioned control law can be directly applied. On the other hand, if internal velocity control of the base is desired, an additional step has to be taken, as will be explained in the following.

\section{Admittance interface for the internally velocity-controlled base}

It is not uncommon for aerial manipulators to have the UAV base independently velocity controlled (e.g., [24]). Either due to the use of commercially available autopilots or to the application of velocity-controlled actuators, as the winches of the SAM [10]. In such cases, the controller presented in the previous section has to be adapted in order to become applicable to such hybrid systems.

Since this issue also appears in the field of mobile robots, a solution has been proposed in [25] and can be suitably adapted to aerial manipulators. The proposed solution relies on adding an admittance interface, which receives the commanded torques produced by the hierarchical controller as the input of a virtual dynamical system, whose velocity is then given as reference to the velocity controller of the base. For that purpose, it is assumed that the controller of the base is a high-gain controller, which can perfectly follow the desired reference.

For the present application, one can decompose the vector of local generalized coordinates as $\boldsymbol{q}=\left[\begin{array}{ll}q_{b} & \boldsymbol{q}_{m}^{T}\end{array}\right]^{T} \in \mathbb{R}^{8}$, where $q_{b} \in \mathbb{R}$ is the yaw joint and $\boldsymbol{q}_{m} \in \mathbb{R}^{7}$ corresponds to the manipulator joints. Similarly, the generalized torques can be decomposed as $\tau=\left[\begin{array}{ll}\tau_{b} & \tau_{m}^{T}\end{array}\right]^{T} \in \mathbb{R}^{8}$. In order to determine desired values for $q_{b}$ from the torque commands generated by the controller defined in (13)-(14), the following admittance interface can be implemented.

$$
M_{a d m} \ddot{q}_{b, d e s}+D_{a d m} \dot{q}_{b, d e s}=\tau_{b},
$$

where $M_{a d m} \in \mathbb{R}$ and $D_{a d m} \in \mathbb{R}$ are positive inertia and damping values, respectively. This interface allows for the computation (via integration) of $\dot{q}_{b, \text { des }}$. Therefore, under the assumption of having a high-gain velocity controller, which implies that $\dot{q}_{b} \approx \dot{q}_{b, \text { des }}$ and that the dynamic interference of the manipulator on the base motion is negligible [25], the dynamics of the aerial system in generalized coordinates becomes

$$
\begin{aligned}
& {\left[\begin{array}{cc}
M_{a d m} & \mathbf{0} \\
\boldsymbol{M}_{b m}(\boldsymbol{q}) & \boldsymbol{M}_{m}(\boldsymbol{q})
\end{array}\right]\left[\begin{array}{c}
\ddot{q}_{b} \\
\ddot{\boldsymbol{q}}_{m}
\end{array}\right]+\left[\begin{array}{cc}
D_{a d m} & \mathbf{0} \\
\boldsymbol{C}_{b m}(\boldsymbol{q}) & \boldsymbol{C}_{m}(\boldsymbol{q})
\end{array}\right]\left[\begin{array}{c}
\dot{q}_{b} \\
\dot{\boldsymbol{q}}_{m}
\end{array}\right]+} \\
& {\left[\begin{array}{c}
0 \\
\boldsymbol{G}_{m}(\boldsymbol{q})
\end{array}\right]=\tau+\tau_{\text {ext }},}
\end{aligned}
$$

where $M_{b m}$ and $\boldsymbol{C}_{b m}$ are the inertia and Coriolis couplings between the base and the manipulator. As suggested in [25], 
in order to achieve completely decoupled dynamics between base and manipulator, an extra term $\tau_{\text {comp }}$ can be added to the control law, which cancels out the effects of $\boldsymbol{M}_{b m}$ and $\boldsymbol{C}_{b m}$ using the values of $\ddot{q}_{b, d e s}$ and $\dot{q}_{b, d e s}$, computed through (21).

Having defined the system dynamics and the compensation action, the control law presented in Section III-B can be applied considering the dynamics in (22) instead of (1) and adding $\tau_{\text {comp }}$ to $\tau$ in (13).

As mentioned in [10], the SAM is able to directly exert body wrenches commanded by the controller through the propellers. However, in order to prepare the system for a possibly hybrid velocity-torque control strategy, the yaw controller presented in that paper was applied as an innerloop velocity controller. The values of $M_{a d m}$ and $D_{a d m}$ were manually tuned such that the inner-loop controller could be considered as a high-gain controller.

\section{EXPERIMENTAL VALIDATION}

For the validation of the proposed framework, the SAM (Fig. 2) was employed. Three sets of experiments were carried out. The first one aims at confirming that the hierarchical-control strategy presented in the previous sections meets its desired goal, namely, enabling the motion of the flying base in the null space of the end-effector. This first step takes into account the case where the base is torquecontrolled by directly applying the approach presented in Section III-B and also the case of a velocity-controlled base by adding the admittance interface presented in Section IIIC. The second validation task is more goal-oriented, where the framework was applied to overcome an occlusion caused by the arm. Finally, the proposed framework was applied to a real outdoor peg-in-hole scenario. The results of the three validation tasks are shown in the following subsections.

\section{A. Quantitative validation}

The first validation experiments aim at analyzing the motion decoupling capabilities of the approach for both torque and velocity-controlled flying bases. In order to do so, the base is commanded to a desired orientation while the end-effector is required to be steady.

Initially, the case of force-controlled aerial manipulators was analyzed. For that, the controller presented in Section III-B was applied to the system for keeping the manipulator steady as the primary task and moving the base by 50 degrees around its suspension axis as the secondary one. The results of that application are depicted in Fig. 3. Fig. 3a shows the orientation of the base in Roll-Pitch-Yaw (RPY) angles. The dashed black line on the bottom plot represent the desired yaw motion while the red lines on the three plots show the orientation of the platform, measured by an inertial measurement unit (IMU). It can be seen that the flying platform was able to regulate to the desired orientation within around 5 seconds. The top and middle plots show that roll and pitch deviations remained within a range of less than one degree during that motion, therefore validating the initial idea of controlling the platform only in yaw.
Figs. $3 \mathrm{~b}$ and $3 \mathrm{c}$ depict the position and orientation of the end-effector as retrieved by the IMU and joint-angle measurements, and the forward kinematics of the manipulator. It can be seem that, after an initial jump of around $3 \mathrm{~cm}$ in the $\mathrm{x}$-axis and smaller amplitudes in $\mathrm{y}$ and $\mathrm{z}$, the positions of the end-effector remained close the desired ones. In Fig. $3 c$ a transient deviation of less than 0.2 degrees was observed in yaw, while roll and pitch deviations remained close to zero. The reason for having non-zero steady-state errors, especially in end-effector positions, is most likely due to imperfect gravity compensation, which affects the arm in different ways according to its configuration. On the other hand, the deviations observed during the convergence period of the platform are due to inaccuracies in the dynamic model, which prevent the system from a complete dynamic decoupling. Nevertheless, such deviations should not prevent common aerial manipulation tasks to be fulfilled.

Subsequently, the same task was performed by the SAM, but this time an admittance interface was used in order to provide the inner-loop yaw controller from [10] with desired velocities based on the torques commanded by the wholebody control law. $M_{a d m}$ and $D_{a d m}$ were tuned beforehand to generate slow enough velocities, such that the perfect tracking assumption would hold for the yaw controller. The chosen values were $M_{a d m}=7.2 \mathrm{~kg} \mathrm{~m}^{2}$ and $D_{a d m}=64 \mathrm{~kg} \mathrm{~m}^{2} / \mathrm{s}$.

The results of that application can be seen in Fig. 4. Fig. $4 \mathrm{a}$ depicts the RPY angles of the platform during the task. It can be seen that desired yaw pose was achieved in a smoother, but slower manner compared to the force controlled platform, due to the low-pass filter characteristic of the admittance interface, as can be noted from (21). That behavior may or may not be desired, depending on the task being performed. The limiting factor for the SAM was ensuring tracking capabilities of the yaw controller. Moreover, it can be noted that the pitch and roll-angle deviations presented similar magnitudes to the previous case.

In Fig. 4b, larger deviations can be observed compared to Fig. 3b. That is most likely because the transient response took longer than in the previous case. Therefore, although a complete decoupling between the two tasks is theoretically guaranteed by the controller presented in Section III, that result relies on perfect knowledge of the system dynamics, which is hard to achieve. Therefore, the maximum transient deviation in position was $5 \mathrm{~cm}$, with a steady-state deviation of $2 \mathrm{~cm}$ along the $\mathrm{y}$-axis, due to imperfect gravity compensation. Moreover, in Fig. 3c larger deviations were also observed in the orientation of the end-effector during the transient response (around 0.3 degrees in yaw and less than that in roll and pitch). Such deviations can be reduced by deriving a more accurate dynamic model, if needed.

When comparing the two approaches, one might argue that the force-based hierarchical control yields better results than the velocity-based one. Unless necessary, it is advisable to let the controller directly command base forces, if that option is available, in order not to unnecessarily increase the complexity of the problem being solved. Nevertheless, it has been shown that the introduction of an admittance interface 
also allowed the hierarchical decoupling task to be performed for a velocity-controlled base. Therefore, the application of the framework presented in this paper is not limited to aerial robots with force-controlled bases, but also extends to cases when an inner-loop velocity controller is present.

\section{B. Qualitative validation}

After showing the motion-decoupling capabilities of the hierarchical controller, another set of experiments for validating its effectiveness in overcoming occlusions of the onboard camera was performed. For that purpose, the robot was intentionally set to a configuration where both a marker and a hole of the same box used in [9] for a peg-in-hole task were occluded and, consequently, vision-aided teleoperation could not be performed. Since the performance of the velocitycontrolled base was shown to be slightly worse than the torque-controlled one, it was chosen for this validation step. It is reasonable to assume that, if the task can be fulfilled with the controller with lower performance, it can also be fulfilled with a better one.

The results are demonstrated by the sequence of images shown in Fig. 5. The system starts at a configuration where both the marker and the hole are occluded (see Figs. 5a and 5b). Subsequently, the proposed approach is applied in order to move the base to a pose where the FoV is more favorable (Figs. 5c and 5d). It can be seen that the base motion happened without disturbing the end-effector, which kept the same Cartesian pose (compare Figs. 5a and 5c).

With that result, it is verified that proposed framework is able to solve the problem of a poor FoV. Therefore, the only step left was to apply it to a real scenario, as will be seen in the following subsection.

\section{Peg-in-hole experiments}

The last part of the experimental evaluation was carried out in an outdoor scenario, where the SAM, hanging from a mobile crane, was teleoperated in order to accomplish a peg-in-hole task in a high and confined environment using the scheme shown in Fig. 1.

During that task, the on-board camera lost sight of the hole due to coupled commands between DoFs, i.e., since the same input device was used to command different DoFs of the platform, the operator unintentionally moved the base while commanding another task. The configuration of the platform and the camera view during that moment are shown in Figs. $6 a$ and $6 b$, respectively. At that point, the task would have to be aborted if no FoV adjustment were possible. However, using the proposed framework, the operator was able to command the base to a more favorable pose and finally complete the peg-in-hole task (see Figs. 6c and 6d).

In addition to the previously mentioned task images, a set of plots that show the performance of the system are provided in Fig. 7. Fig 7a shows the desired yaw commanded by the operator as well as the RPY values measured by the IMU. There, the moments where the yaw angle is accidentally commanded to a poor FoV and the command for regaining a good view of the task are captured at $t \approx 50 \mathrm{~s}$ and $t \approx 90 \mathrm{~s}$, respectively. Despite some vibrations (which were not present indoors), the yaw tracking capabilities of the hierarchical controller are proved satisfactory. It can also be seen that the roll and pitch values are kept around zero, as in the indoor evaluation, which supports the assumption of no motion along those axes.

Figs. 7b and Fig.7c show the values of the relative position and orientation (absolute angle) of the end-effector with respect to the hole, computed by the visual-inertial algorithm presented in [9]. The dashed vertical line at $t \approx 190 \mathrm{~s}$ marks the moment where the peg is inserted into the hole. At that moment, the relative $\mathrm{x}-\mathrm{y}$ position and angle are close to zero and the relative z-position is crossing zero, i.e., the endeffector is aligned with hole and moving into it.

The results presented in this section demonstrate the applicability of the proposed framework to real scenarios where the motion of the flying base is exploited in order to assist the operator in fulfilling a desired task, which would otherwise have to be aborted in case of occlusion or loss of sight.

\section{CONClusions AND Future Work}

This paper validated the application of hierarchical wholebody control to achieve decoupled motion of the flying base and avoid occlusions and loss of sight of the on-board camera. Both quantitative and qualitative validation experiments were performed, where the base was commanded to turn, as a secondary task, while the end-effector was commanded to keep its pose, as a primary task. In addition, an outdoor pegin-hole task demonstrated the efficacy of the approach in real scenarios. With that, the need for a such a controller and its contribution to successful vision-based telemanipulation and autonomous manipulation was demonstrated.

The limitations of the proposed approach lie in the fact that, despite not needing exact knowledge of the system dynamics for guaranteeing stability, its performance is degraded in case the model is inaccurate. Moreover, the requirement of full row rank of the Jacobian limit the convergence and stability properties to hold only locally. Applying specific techniques for overcoming those issues is left as future work.

The future of this work will be divided into two directions. The first direction will be extending it to cope with the winch actuators and also to allow for different configurations of the base away from its zero roll-pitch and zero $x-y$ pose. The second direction will focus on autonomously commanding the position of the flying base in order to optimize the field of view and allowing for blended commands between autonomy and human operator in a shared-control fashion. 

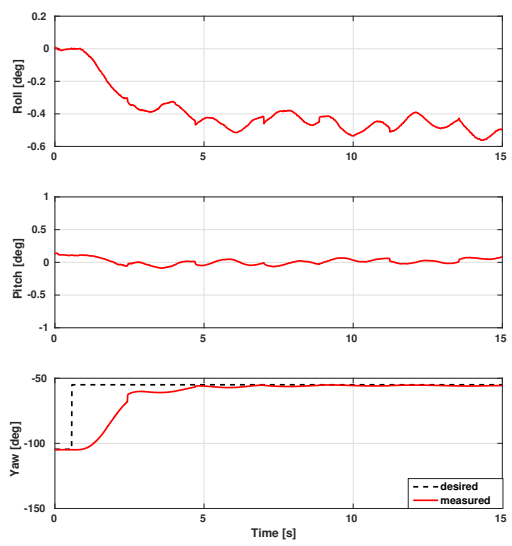

(a) Base orientation in RPY.
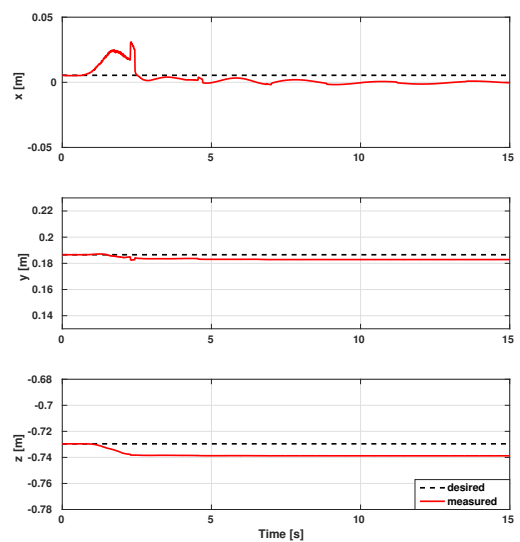

(b) End-effector position.
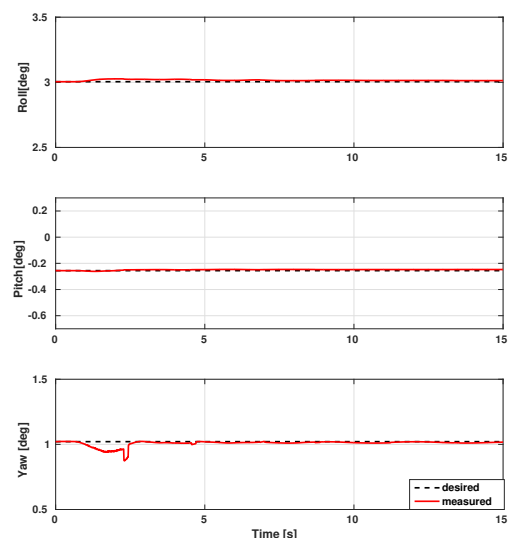

(c) End-effector orientation in RPY.

Fig. 3: Position and Orientation plots with force-controlled base.
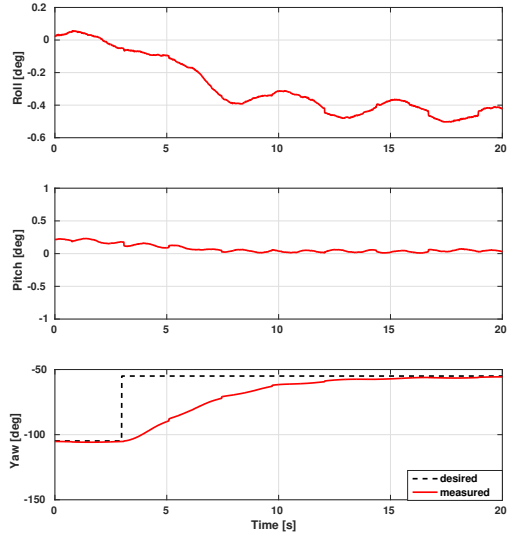

(a) Base orientation in RPY.
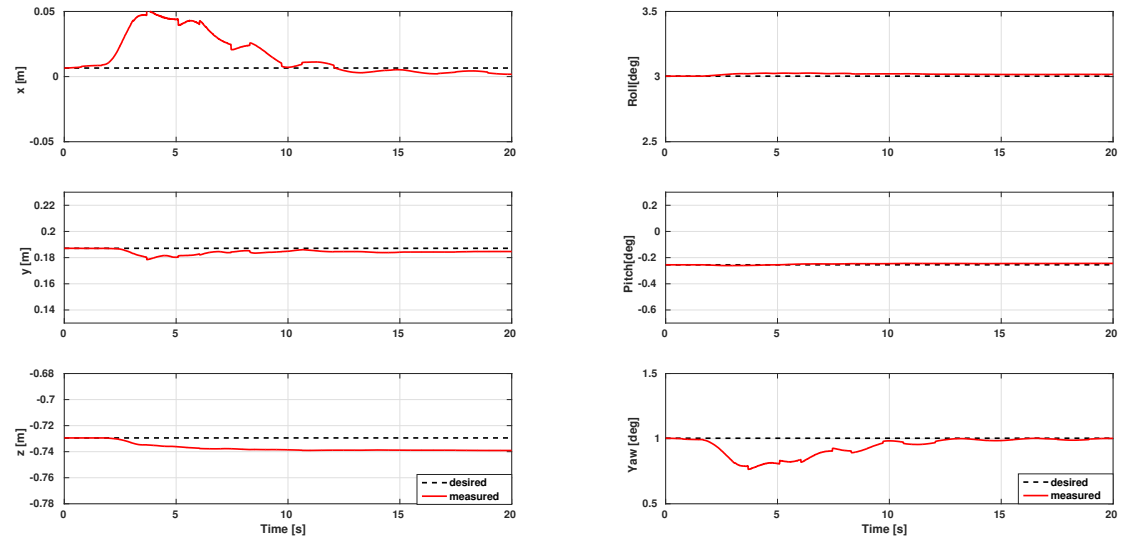

(b) End-effector position.

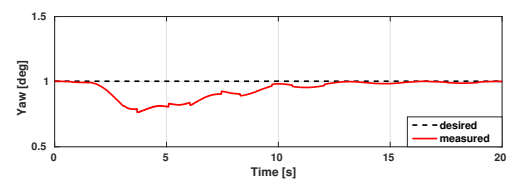

(c) End-effector orientation in RPY.

Fig. 4: Position and Orientation plots with velocity-controlled base through admittance interface.

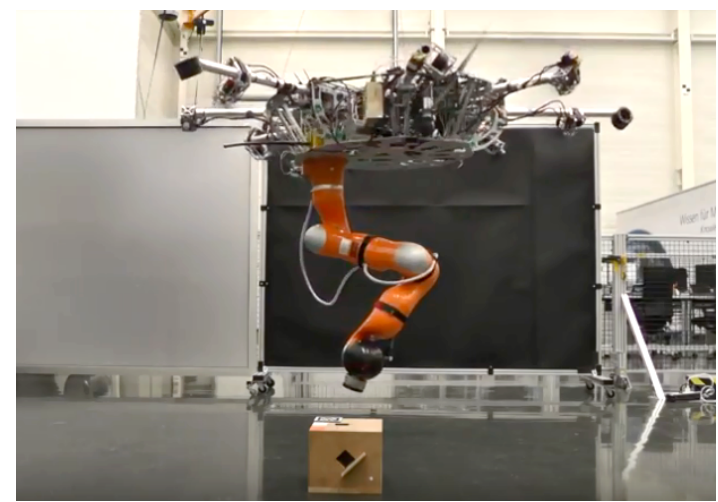

(a)

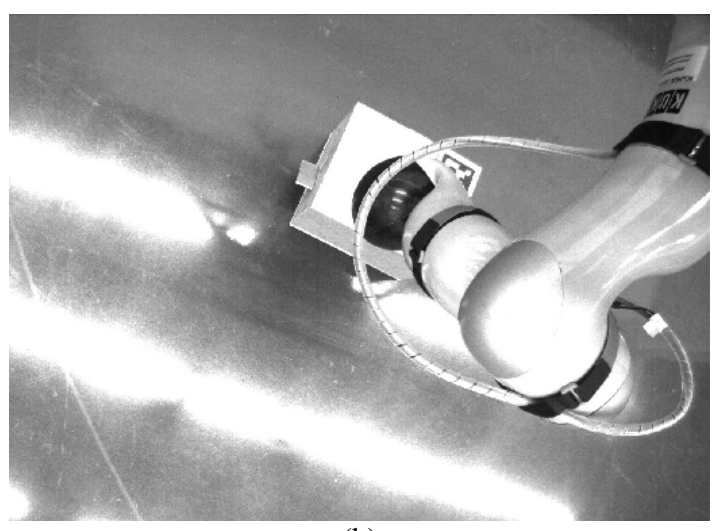

(b) 


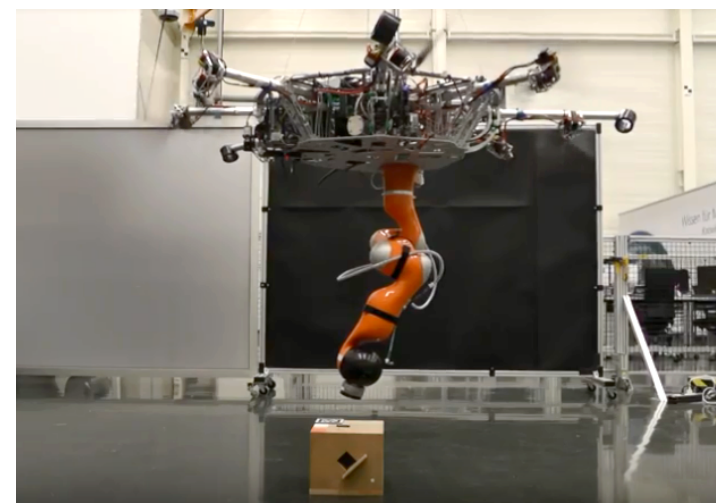

(c)
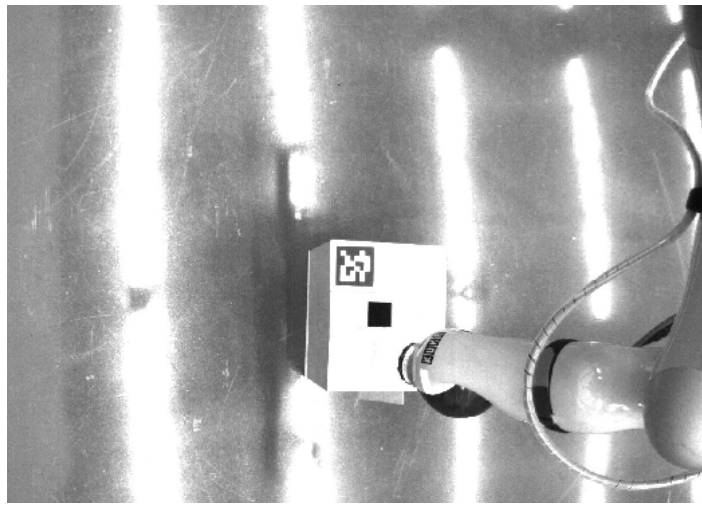

(d)

Fig. 5: External images (left) and images from eye-to-hand camera (right). (a,b): Marker and hole are occluded. (c,d): The proposed approach is used to command the base to a pose where the marker and the hole are visible while the end-effector remains static.

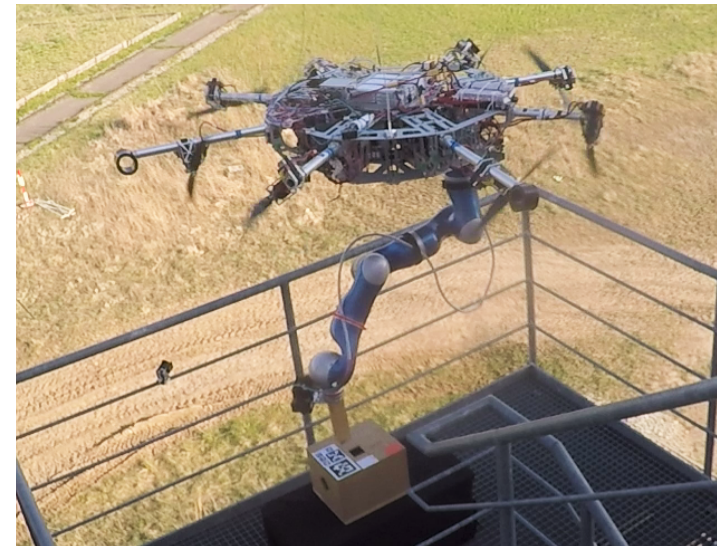

(a)

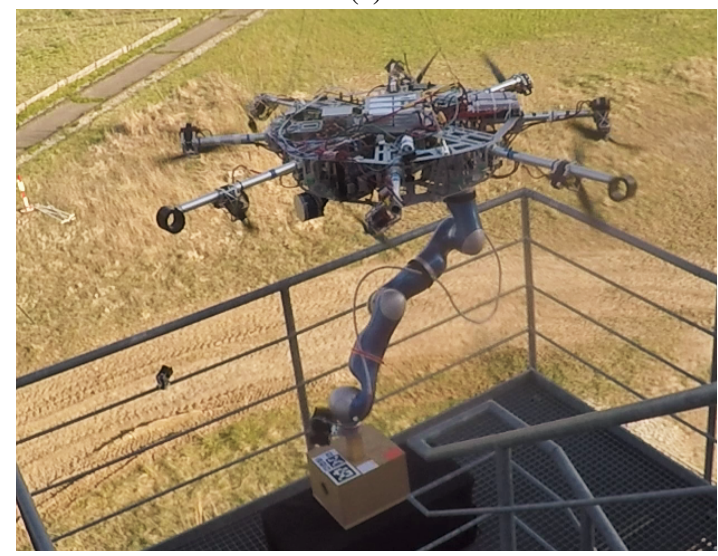

(c)

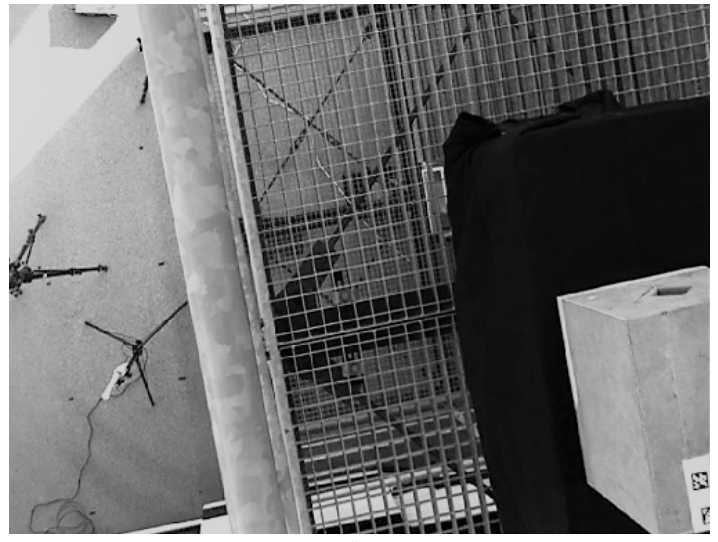

(b)

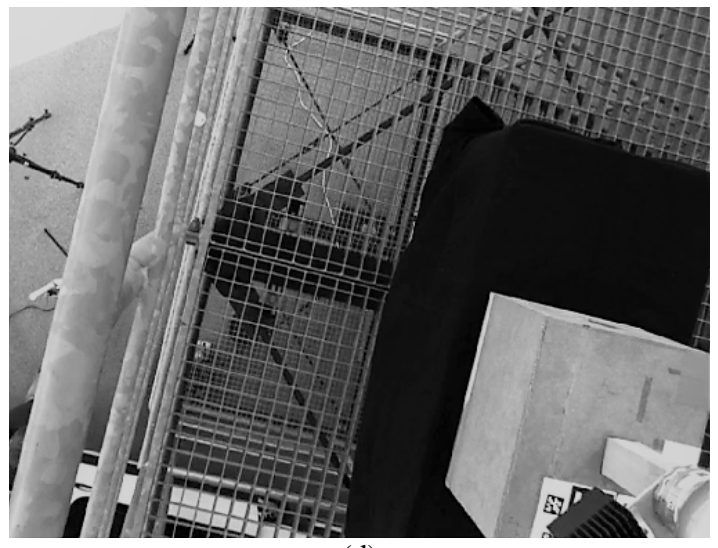

(d)

Fig. 6: External images (left) and images from eye-to-hand camera (bottom). (a,b): Neither the hole nor the peg are visible. $(\mathrm{c}, \mathrm{d})$ : After null-space motion of the base, the hole became visible and the peg-in-hole task could be accomplished. 


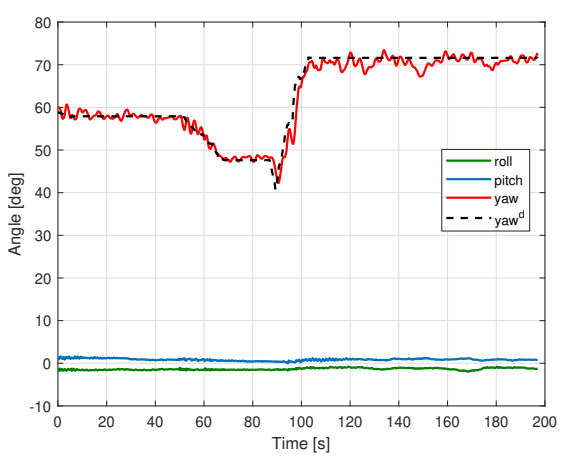

(a) Base orientation in RPY.

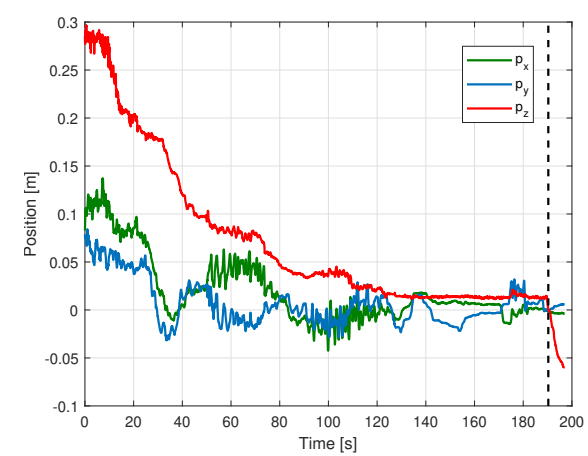

(b) End-effector to target position.

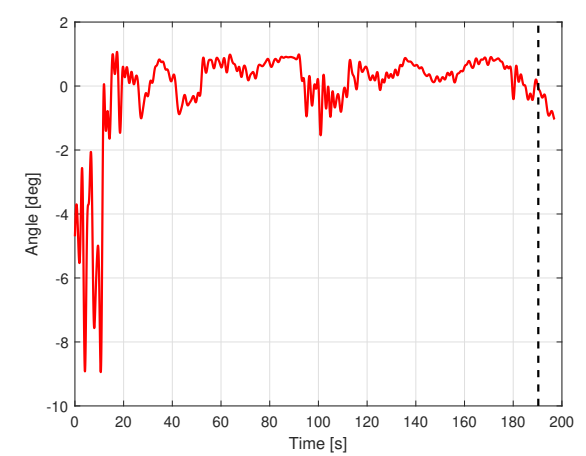

(c) End-effector to target orientation.

Fig. 7: Position and Orientation plots for the peg-in-hole task.

\section{REFERENCES}

[1] N. Pouliot, P.-L. Richard, and S. Montambault, "Linescout technology opens the way to robotic inspection and maintenance of high-voltage power lines," IEEE Power and Energy Technology Systems Journal, vol. 2, no. 1, pp. 1-11, 2015.

[2] L. Matikainen, M. Lehtomäki, E. Ahokas, J. Hyyppä, M. Karjalainen, A. Jakkkola, A. Kukko, and T. Heinonen, "Remote sensing methods for power line corridor surveys," ISPRS Journal of Photogrammetry and Remote Sensing, vol. 119, pp. 10-31, 2016.

[3] A. Jimenez-Cano, G. Heredia, and A. Ollero, "Aerial manipulator with a compliant arm for bridge inspection," in 2017 International Conference on Unmanned Aircraft Systems (ICUAS). IEEE, 2017, pp. 1217-1222.

[4] A. E. Jimenez-Cano, P. J. Sanchez-Cuevas, P. Grau, A. Ollero, and G. Heredia, "Contact-based bridge inspection multirotors: Design, modeling, and control considering the ceiling effect," IEEE Robotics and Automation Letters, vol. 4, no. 4, pp. 3561-3568, 2019.

[5] M. Tognon, H. A. T. Chávez, E. Gasparin, Q. Sablé, D. Bicego, A. Mallet, M. Lany, G. Santi, B. Revaz, J. Cortés et al., "A trulyredundant aerial manipulator system with application to push-and-slide inspection in industrial plants," IEEE Robotics and Automation Letters, vol. 4, no. 2, pp. 1846-1851, 2019.

[6] P. Ramon-Soria, B. C. Arrue, and A. Ollero, "Grasp planning and visual servoing for an outdoors aerial dual manipulator," Engineering, vol. 6 , no. 1 , pp. $77-88,2020$.

[7] M. Mohammadi, A. Franchi, D. Barcelli, and D. Prattichizzo, "Cooperative aerial tele-manipulation with haptic feedback," in 2016 IEEE/RSJ International Conference on Intelligent Robots and Systems (IROS). IEEE, 2016, pp. 5092-5098.

[8] A. Ollero, G. Heredia, A. Franchi, G. Antonelli, K. Kondak, A. Sanfeliu, A. Viguria, J. R. Martinez-de Dios, F. Pierri, J. Cortes, A. Santamaria-Navarro, M. A. Trujillo Soto, R. Balachandran, J. Andrade-Cetto, and A. Rodriguez, "The aeroarms project: Aerial robots with advanced manipulation capabilities for inspection and maintenance," IEEE Robotics Automation Magazine, vol. 25, no. 4, pp. 12-23, Dec 2018.

[9] J. Lee, R. Balachandran, Y. Sarkisov, M. De Stefano, A. Coelho, K. Shinde, M. J. Kim, R. Triebel, and K. Kondak, "Visual-inertial telepresence for aerial manipulation," in 2020 IEEE International Conference on Robotics and Automation (ICRA), 2020, pp. 12221229.

[10] Y. S. Sarkisov, M. J. Kim, D. Bicego, D. Tsetserukou, C. Ott, A. Franchi, and K. Kondak, "Development of sam: cable-suspended aerial manipulator," in IEEE International Conference on Robotics and Automation (ICRA), 2019.

[11] A. Dietrich, C. Ott, and A. Albu-Schäffer, "An overview of null space projections for redundant, torque-controlled robots," The International Journal of Robotics Research, vol. 34, no. 11, pp. 1385-1400, 2015.

[12] V. Lippiello, J. Cacace, A. Santamaria-Navarro, J. Andrade-Cetto, M. A. Trujillo, Y. R. Esteves, and A. Viguria, "Hybrid visual servoing with hierarchical task composition for aerial manipulation," IEEE Robotics and Automation Letters, vol. 1, no. 1, pp. 259-266, 2015.

[13] A. Santamaria-Navarro, P. Grosch, V. Lippiello, J. Solà, and J. Andrade-Cetto, "Uncalibrated visual servo for unmanned aerial manipulation," IEEE/ASME Transactions on Mechatronics, vol. 22, no. 4, pp. 1610-1621, 2017.

[14] F. Huber, K. Kondak, K. Krieger, D. Sommer, M. Schwarzbach, M. Laiacker, I. Kossyk, S. Parusel, S. Haddadin, and A. AlbuSchäffer, "First analysis and experiments in aerial manipulation using fully actuated redundant robot arm," in 2013 IEEE/RSJ International Conference on Intelligent Robots and Systems. IEEE, 2013, pp. 34523457.

[15] J. Park, W. Chung, and Y. Youm, "On dynamical decoupling of kinematically redundant manipulators," in Proceedings 1999 IEEE/RSJ International Conference on Intelligent Robots and Systems. Human and Environment Friendly Robots with High Intelligence and Emotional Quotients (Cat. No. 99CH36289), vol. 3. IEEE, 1999, pp. $1495-1500$.

[16] C. Gabellieri, Y. S. Sarkisov, A. Coelho, L. Pallottino, K. Kondak, and M. J. Kim, "Compliance control of a cable-suspended aerial manipulator using hierarchical control framework," in IEEE/RSJ International Conference on Intelligent Robots and Systems (Accepted), 2020.

[17] A. Coelho, H. Singh, K. Kondak, and C. Ott, "Whole-body bilateral teleoperation of a redundant aerial manipulator," in 2020 IEEE International Conference on Robotics and Automation (ICRA), 2020, pp. 9150-9156.

[18] A. Coelho, Y. Sarkisov, X. Wu, H. Mishra, H. Singh, A. Dietrich, A. Franchi, K. Kondak, and C. Ott, "Whole-body teleoperation and shared control of redundant robots with applications to aerial manipulation," Journal of Intelligent \& Robotic Systems, vol. 102, no. 1, Apr. 2021. [Online]. Available: https://doi.org/10.1007/s10846021-01365-7

[19] Y. Sarkisov, M. J. Kim, A. Coelho, D. Tsetserukou, C. Ott, and K. Kondak, "Optimal oscillation damping control of cable-suspended aerial manipulator with a single imu sensor," in 2020 IEEE International Conference on Robotics and Automation (ICRA), 2020, pp. 53495355.

[20] A. Dietrich and C. Ott, "Hierarchical impedance-based tracking control of kinematically redundant robots," IEEE Transactions on Robotics, vol. 36, no. 1, pp. 204-221, Feb 2020.

[21] O. Khatib, "A unified approach for motion and force control of robot manipulators: The operational space formulation," IEEE Journal on Robotics and Automation, vol. 3, no. 1, pp. 43-53, 1987.

[22] S. Zhang and E. D. Fasse, "Spatial compliance modeling using a quaternion-based potential function method," Multibody System Dynamics, vol. 4, no. 1, pp. 75-101, 2000.

[23] C. Ott, M. A. Roa, and G. Hirzinger, "Posture and balance control for biped robots based on contact force optimization," in 2011 11th IEEE-RAS International Conference on Humanoid Robots, Oct 2011, pp. 26-33.

[24] K. Kondak, F. Huber, M. Schwarzbach, M. Laiacker, D. Sommer, M. Bejar, and A. Ollero, "Aerial manipulation robot composed of an autonomous helicopter and a 7 degrees of freedom industrial manipulator," in 2014 IEEE International Conference on Robotics and Automation (ICRA). IEEE, 2014, pp. 2107-2112.

[25] A. Dietrich, K. Bussmann, F. Petit, P. Kotyczka, C. Ott, B. Lohmann, and A. Albu-Schäffer, "Whole-body impedance control of wheeled mobile manipulators," Autonomous Robots, vol. 40, no. 3, pp. 505517, 2016. 